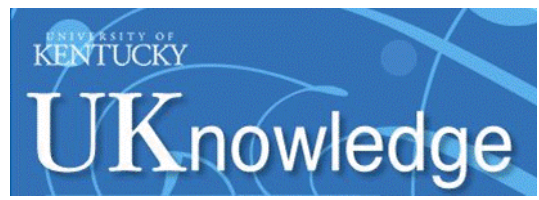

University of Kentucky

UKnowledge

Biosystems and Agricultural Engineering Faculty Publications

$5-21-2018$

\title{
A Low-Cost Method for Collecting Hyperspectral Measurements from a Small Unmanned Aircraft System
}

\author{
Ali Hamidisepehr \\ University of Kentucky, ali.hamidisepehr@gmail.com \\ Michael P. Sama \\ University of Kentucky, michael.sama@uky.edu
}

Follow this and additional works at: https://uknowledge.uky.edu/bae_facpub

Part of the Bioresource and Agricultural Engineering Commons

Right click to open a feedback form in a new tab to let us know how this document benefits you.

\section{Repository Citation}

Hamidisepehr, Ali and Sama, Michael P., "A Low-Cost Method for Collecting Hyperspectral Measurements from a Small Unmanned Aircraft System" (2018). Biosystems and Agricultural Engineering Faculty Publications. 228.

https://uknowledge.uky.edu/bae_facpub/228

This Conference Proceeding is brought to you for free and open access by the Biosystems and Agricultural Engineering at UKnowledge. It has been accepted for inclusion in Biosystems and Agricultural Engineering Faculty Publications by an authorized administrator of UKnowledge. For more information, please contact UKnowledge@lsv.uky.edu. 


\title{
A Low-Cost Method for Collecting Hyperspectral Measurements from a Small Unmanned Aircraft System
}

\author{
Digital Object Identifier (DOI) \\ https://doi.org/10.1117/12.2305934
}

\section{Notes/Citation Information}

Published in Proceedings of SPIE, v. 10664, Autonomous Air and Ground Sensing Systems for Agricultural Optimization and Phenotyping III, article 106640H, p. 1-15.

(C) 2018 SPIE One print or electronic copy may be made for personal use only. Systematic reproduction and distribution, duplication of any material in this paper for a fee or for commercial purposes, or modification of the content of the paper are prohibited.

Ali Hamidisepehra and Michael P. Sama, "A low-cost method for collecting hyperspectral measurements from a small unmanned aircraft system," Proc. SPIE 10664, Autonomous Air and Ground Sensing Systems for Agricultural Optimization and Phenotyping III, 10664H (May 21, 2018). DOI: https://doi.org/10.1117/ 12.2305934

The copyright holder has granted the permission for posting the article here. 


\section{A low-cost method for collecting hyperspectral measurements from a small unmanned aircraft system}

Ali Hamidisepehr, Michael P. Sama

Ali Hamidisepehr, Michael P. Sama, "A low-cost method for collecting hyperspectral measurements from a small unmanned aircraft system," Proc. SPIE 10664, Autonomous Air and Ground Sensing Systems for Agricultural Optimization and Phenotyping III, 106640H (21 May 2018); doi:

10.1117/12.2305934

Event: SPIE Commercial + Scientific Sensing and Imaging, 2018, Orlando, Florida, United States 


\title{
A Low-cost Method for Collecting Hyperspectral Measurements from a Small Unmanned Aircraft System
}

\author{
Ali Hamidisepehr ${ }^{\mathrm{a}}$, Michael P. Sama*a \\ ${ }^{a}$ Department of Biosystems and Agricultural Engineering, University of Kentucky, Lexington, \\ Kentucky, USA 40546-0276; phone:859-218-4325; e-mail: michael.sama@uky.edu
}

\begin{abstract}
Small unmanned aircraft systems (UAS) are a relatively new tool for collecting remote sensing data at dense spatial and temporal resolutions. This study aimed to develop a spectral measurement platform for deployment on a UAS for quantifying and delineating moisture zones within an agricultural landscape. A series of portable spectrometers covering ultraviolet (UV), visible (VIS), and near-infrared (NIR) wavelengths were instrumented using a Raspberry Pi embedded computer that was programmed to interface with the UAS autopilot for autonomous data acquisition. A second set of identical spectrometers were fitted with calibrated irradiance lenses to capture ambient light during data acquisition. Data were collected during the 2017 Great American Eclipse while observing a reflectance target to determine the ability to compensate for ambient light conditions. A calibration routine was developed that scaled raw reflectance data by sensor integration time and ambient light energy. The resulting calibrated reflectance exhibited a consistent spectral profile and average intensity across a wide range of ambient light conditions. Results indicated the potential for mitigating the effect of ambient light when passively measuring reflectance on a portable spectral measurement system. Future work will use multiple reflectance targets to test the ability to classify targets based on spectral signatures under a wide range of ambient light conditions.
\end{abstract}

Keywords: Remote sensing, Ambient light calibration, Unmanned aircraft system, Spectroscopy, Eclipse

\section{Introduction}

Efficient irrigation management is one of the most important issues producers face in arid and semi-arid areas [1-2]. Novel technologies, such as variable-rate irrigation, help to control water usage and result in more efficient irrigation [34]. To spatially implement variable-rate irrigation, a prescription map containing information about the actual water status of the field is needed [5]. Remote sensing is one possible method for obtaining water status in a field and generating near real-time prescription maps for irrigation. Traditional deployments of remote sensing include satellite and conventional aircraft, but they are limited in terms of cost, temporal resolution, and spatial resolution. Unmanned aircraft systems (UAS), or drones, are relatively new tools for collecting remote sensing in agricultural applications [67]. In one study, traditional methods of remote sensing, including satellites and manned aircraft systems, were compared to UAS. It was concluded that UAS were more cost-effective in fields smaller than five hectares and UAS were determined to provide higher precision data [8].

Reflectance-based remote sensing is a potential method for quantifying soil moisture and delineating moisture management zones. Several studies have focused on using visible, multispectral, or hyperspectral cameras mounted on drones to evaluate crop/soil status by monitoring different index values with a high spatial resolution. Spectral reflectance data collected using UAS are extensively used in research to estimate different soil and crop parameters. Example applications include estimating soil fertility [9], generating vegetation indices [10-11], assessing tree crowns for breeding applications [12], yield estimation [13], plant classification [14], weed detection [15], and controlling herbicide applications [16] are only a few applications of UAS in agricultural domain.

Advances in spectrometer development have led to more portable systems, or micro-spectrometers, that are particularly suitable for UAS deployment due their small size and mass. One study showed that measurements from UAS-deployed micro-spectrometers were highly correlated with ground reference measurements and concluded that the UAS platform could provide a faster and more accurate method for spectral data collection [17]. A subsequent study, where a visible micro-spectrometer was used to measure reflectance, showed that the remote sensing estimations were highly correlated to ground spectral measurements [18]. In another study, a spectrometer coupled with a camera was 
mounted on a UAS to measure radiance values from different targets. The same model spectrometer had previously been used to collect data from a satellite in orbit, and when data collected from these two platforms were compared, it was confirmed that the UAS provided an efficient platform for collecting spectral data [19].

Calibration of hyperspectral measurement systems is a challenge due to the large number of factors that can influence spectral response. For lab-based spectrometry, measurements are taken under controlled light conditions, which cannot be applied with UAS-deployed spectrometers under field condition with frequent changes in ambient light. The empirical line method is one of the common approaches for calibrating hyperspectral images against variable illumination. In this approach, tarps or panels with known relative reflectance are placed in a field during the data collection. By finding the relationship between known reflectance values and digital count output of the sensor, an equation is obtained and then applied to all measurements to complete the calibration process [17-18]. The data collection period is limited since changing sun angle during data acquisition affect the reflectance [9]. Transient cloud cover can also substantially affect the amount of reflected light observed. Another limitation is the practical limitation of having tarps or other reference targets in all images, especially when high resolution data is desired or a large area is covered [20]. Devising a method that can keep track of ambient light changes while measuring the reflectance from a spectral target is desired. By automating this measurement process through ambient light detection, percent reflectance can be obtained for every single wavelength in the spectrum at a low cost and under various ambient light conditions. [21-22].

Previous work by [23] showed that relative reflectance in the visible and near-infrared range could be used to optimally develop a normalized difference water index (NDWI) that predicted soil moisture content from direct soil observations. However, the experiment relied on a controlled light source, which is not practical for UAS-based spectral measurements. In this study, the overall goal was to develop a spectral sensing platform suitable for UAS deployment to measure the reflectance from a reference target to assist with the development of a calibration procedure that is functional over a wide range of ambient light conditions. Specific objectives included:

1. Instrument a series of portable spectrometers and integrate into a UAS for autonomous data collection.

2. Develop a method to compensate for ambient light conditions and sensor integration time when collecting raw spectral reflectance measurements.

\section{Material and Methods}

\subsection{Sensor Instrumentation}

In this experiment, two spectral data acquisition systems were used: an updward-facing ambient light system to measure solar irradience at ground level; and a downward-facing reflectance system to measure reflectance from a target located at ground level. Both systems consist of three spectrometers (STS; Ocean Optics; Dunedin, FL) in the ultraviolet (UV), visible (VIS), and near-infrared (NIR) ranges (Table 1). Ambient light and reflectance raw spectral measurements were reported as 14-bit digital count values in integer format. The ambient light spectrometers were fitted with optical diffusers and factory calibrated in compliance with NIST practices. Calibration data were used to convert raw spectral measurements at each wavelength from an integer count value to units of microjoules. The reflectance spectrometers were fitted with collimating lenses to set the field-of-view (FOV) and align the light entering the spectrometers.

Table 1: Model number and lens type for individual spectrometers in ambient light and reflectance systems.

\begin{tabular}{cccc}
\hline System & Spectrometer Type & Model Number & Optics \\
\hline \multirow{3}{*}{ Ambient light } & UV & STS-UV-L-25-400-SMA & CC-3-DA \\
& VIS & STS-VIS-L-50-400-SMA & CC-3-DA \\
& NIR & STS-NIR-L-25-400-SMA & CC-3-DA \\
\hline \multirow{3}{*}{ Reflectance } & UV & STS-UV-L-100-400-SMA & 74-DA \\
& VIS & STS-VIS-L-100-400-SMA & 74-DA \\
& NIR & STS-NIR-L-100-400-SMA & 74-DA \\
\hline
\end{tabular}




\subsection{Data Acquisition System}

The target application for this measurement system was to automate the collection of spectral reflectance data from a UAS platform while compensating for varying ambient light conditions. This meant data from multiple spectrometers would need to be remotely recorded at defined locations or intervals. To accomplish this requirement, each system was coupled with a Raspberry Pi 3 (RPi) (B V1.2; Raspberry Pi Foundation; Cambridge, United Kingdom) as an embedded data acquisition system to control the measurement process. The spectrometer manufacturer provided a software development kit (SDK) for the RPi that was preinstalled on a Raspbian distribution of Linux (Version 7; Raspberry Pi Foundation, Cambridge, United Kingdom). The SDK configured the RPi as web server, facilitating wireless control of individual spectrometers via a WiFi connection to a PC. Since WiFi control of the UAS-deployed system would not be practical in agricultural applications, a pulse-width-modulation (PWM) to digital converter was used to allow the UAS autopilot to trigger measurements using a digital input on the RPi. The UAS was fitted with an off-the shelf autopilot system (A3; DJI; Nanshan District, Shenzhen, China) and configured to output a PWM signal corresponding to the "shutter" command normally used to trigger the capture of imagery from an on-board camera. As a result, triggering the spectrometer was identical to taking a picture from the perspective of mission planning software. Figure 1 shows the major components used in the reflectance and ambient light systems.

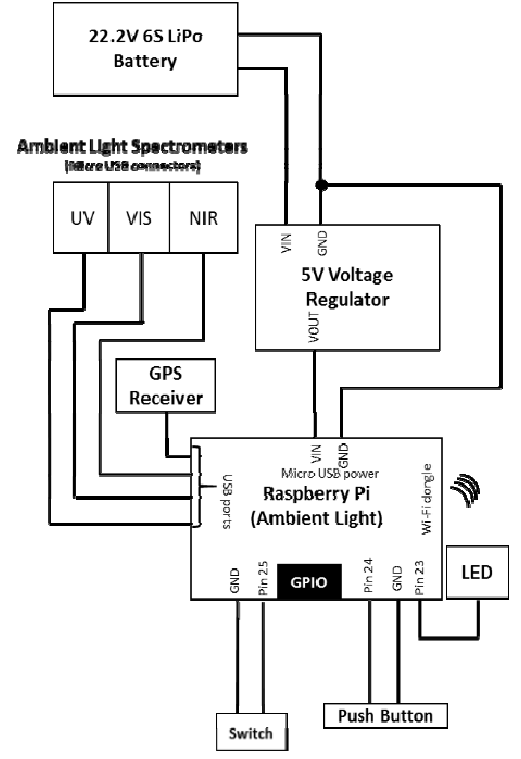

a.

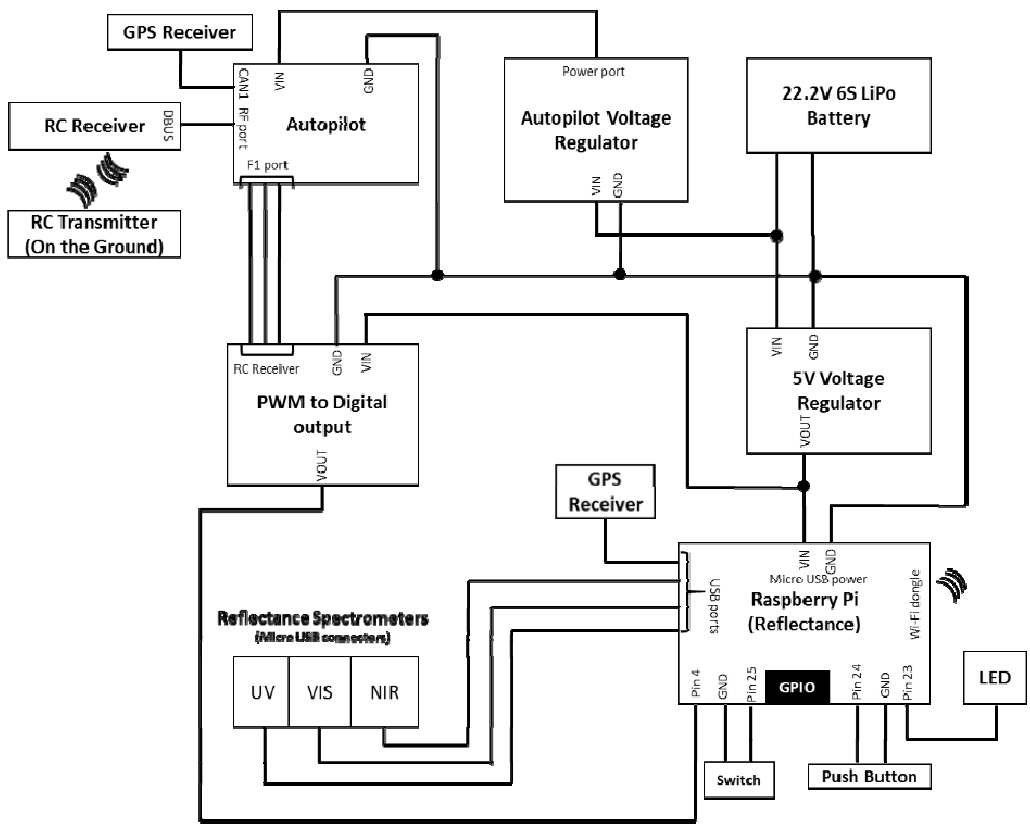

b.

Figure 1: Hardware block diagram schematic of the data acquisition system including all components for a. ambient light and $b$. reflectance measurement systems.

Components inside the autopilot included: a voltage regulator (A300P-PMU PRO, DJI) to regulate input voltage to the autopilot; a GPS receiver (A300P-GPS COMPASS PRO, DJI) to update the time and the location of the UAS; and a radio control (RC) transmitter (GL858A; DJI) and a receiver (R810A; DJI; Nanshan District, Shenzhen, China) to control the UAS manually and also to monitor the flight via a live video feed.

Both ambient light and reflectance systems were equipped with similar supporting components including: a voltage regulator (2858; Pololu; Las Vegas, NV) to set the RPi input voltage at 5 V; a GPS receiver (BU-353S4; USGlobalSat; Chino, CA) with a USB connection to sychrnoize the local time on the RPi to Unversal Coordinated Time (UTC); and two LEDs to indicate if sensors were taking measurement and to show if the system was expecting an external signal to trigger a measurement or if sampling was to proceed automatically on a pre-programmed interval. A PWM-to-digital converter (2801; Pololu; Las Vegas, NV) was used in the reflectance system to allow the autopilot to trigger functions on 
the RPi via a logic level on a digital input pin. For UAS operations, the ambient light system was configured to collect data on a regular interval during UAS deployment, rather than triggering remotely, to remove the need for a wireless trigger between the UAS and ambient light system. For ground operations, both systems were configured to collect data on a regular interval. Ambient light and reflectance spectral data were interpolated to a synchronous time interval prior to compensating reflectance values for ambient light.

Two Python scripts were written and configured to run immediately upon startup. The first script continously polled digital input pins associated with pushbutton switches and the PWM-to-digital converter. Upon receiving the appropriate signal, or in the event the system was configured to take measurements at a predefined interval, the script would generate a series of HTML functions call to the SDK web server causing the spectrometers to take measurements and the RPi to record the data on the onboard SD card. Each measurement produced a new file with a unique filename. Filenames consisted of the spectrometer serial number and a local date/time stamp. The second script initiated the GPSD Linux library that facilitated comminucation with the GPS receiver and regularly updated the local date and time on the RPi to UTC. This was necessary because the RPi does not have a real-time clock for keeping track of local time while powered down and is normally configured to set the local time from a network time protocol (NTP) time server via the internet. Figure 2 and Figure 3 demonstrate the data collection process in each set of spectrometers when operating at a regular interval and through a digital input trigger, respectively. An on-off pushbutton switch mounted to each system was used to determine which process was implemented so that identical programs resided on the ambient light and reflectance systems.

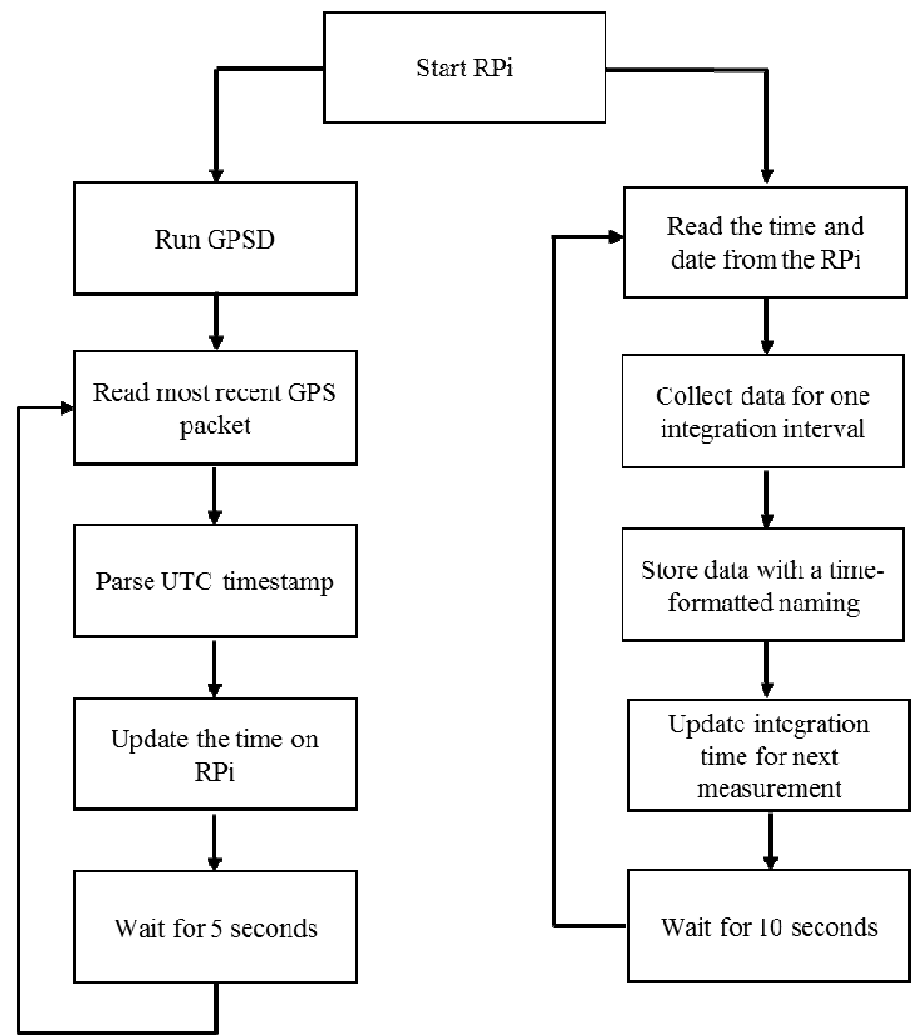

Figure 2: Embedded control and data acquisition software block diagram for the ambient light system. 


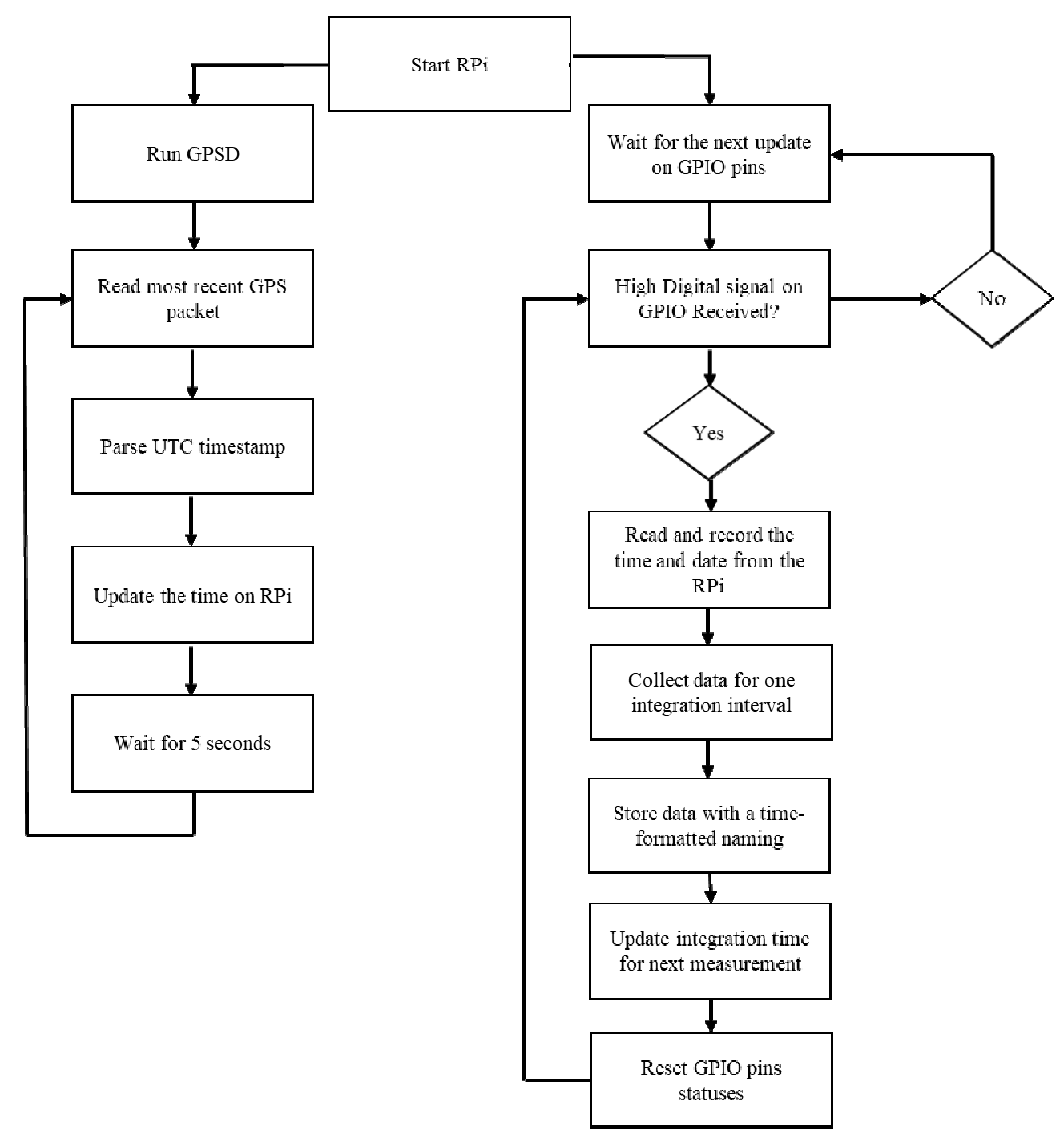

Figure 3: Embedded control and data acquisition software block diagram for the reflectance system.

Each path leaving the StartRPi block represents an individual Python script. In the left path, the UTC timestamp is extracted from the most recent GPS data packet and used to update the time on the RPi at a 5-second interval. The right path differ slightly depending on if the system is intended to be triggered a regular interval based on a timer (Figure 2) or by a change of state on a digital input corresponding to a signal from the PWM-to-digital converter when used with the autopilot (Figure 3). Regardless of the trigger method, the RPi stored the current local time and recorded data from the spectrometers into tab-delimited text files. Figure 4 shows a picture of the reflectance system mounted on a UAS. 


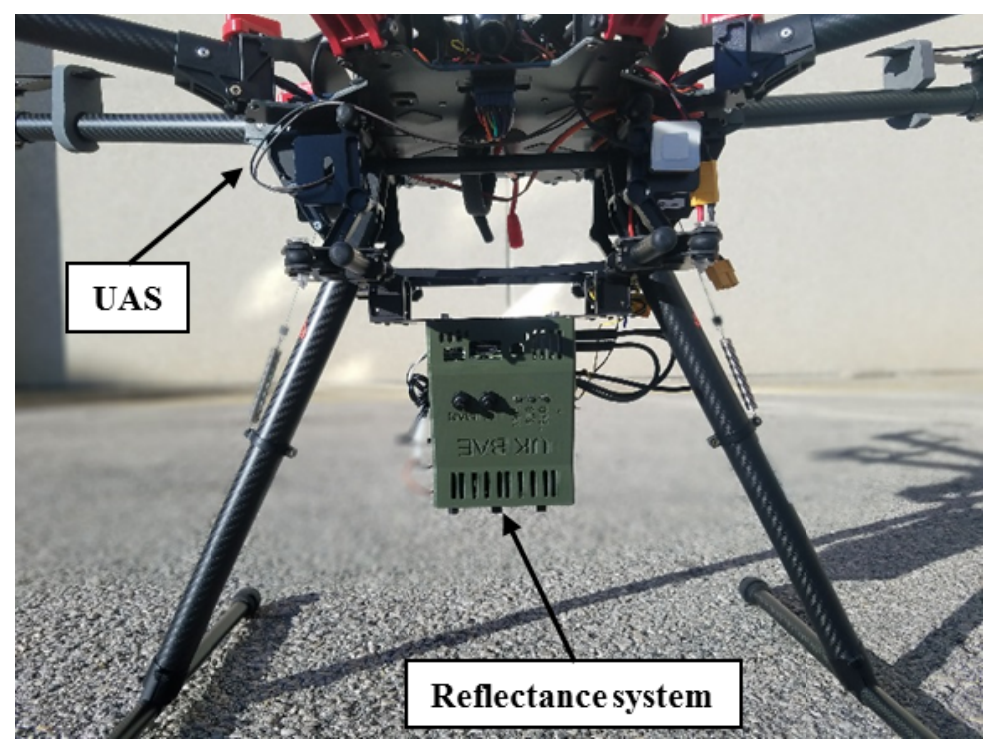

Figure 4: Reflectance system mounted on a DJI S1000+. Data are recorded at pre-defined GNSS waypoints by triggering the shutter command in the UAS autopilot.

\subsection{Reference Target and Test Stand}

A reference target was fabricated and calibrated against a white standard. The reflectance target consisted of a 30.5 $\mathrm{cm} \times 30.5 \mathrm{~cm} \times 1.9 \mathrm{~cm}$ piece of plywood with a $0.08 \mathrm{~cm}$ thick Teflon sheet glued to the surface. A threaded insert was mounted at the center of the reference target to allow the reference target to be mounted to a standard surveying tripod during field use. The reference target was calibrated using a backscatter reflectance system that consisted of: visible and near-infrared spectrometers (HR400-7-VIS-NIR, NIRQuest512, Ocean Optics, Dunedin, Fla.); a tungsten-halogen light source (HL-2000-HP-FHSA, Ocean Optics); a fiber optic backscatter reflectance probe (QR200-12-MIXED, Ocean Optics); and a Spectralon calibration target (WS-1-SL, Ocean Optics). It was assumed that the reflectance target had a constant spectral response at different temperatures and atmospheric conditions.

A test stand was used to consistently position the target underneath the reflectance system. The stand was $1 \mathrm{~m}$ tall (from the top of the stand) and has a square base for locating the reflectance target. Spectrometers were located on the top of the stand above the target and off-center to prevent the middle spectrometer from sampling the threaded insert (Figure 5). The height of reflectance spectrometers can be adjusted for further experiments, and the area sampled will change accordingly. 


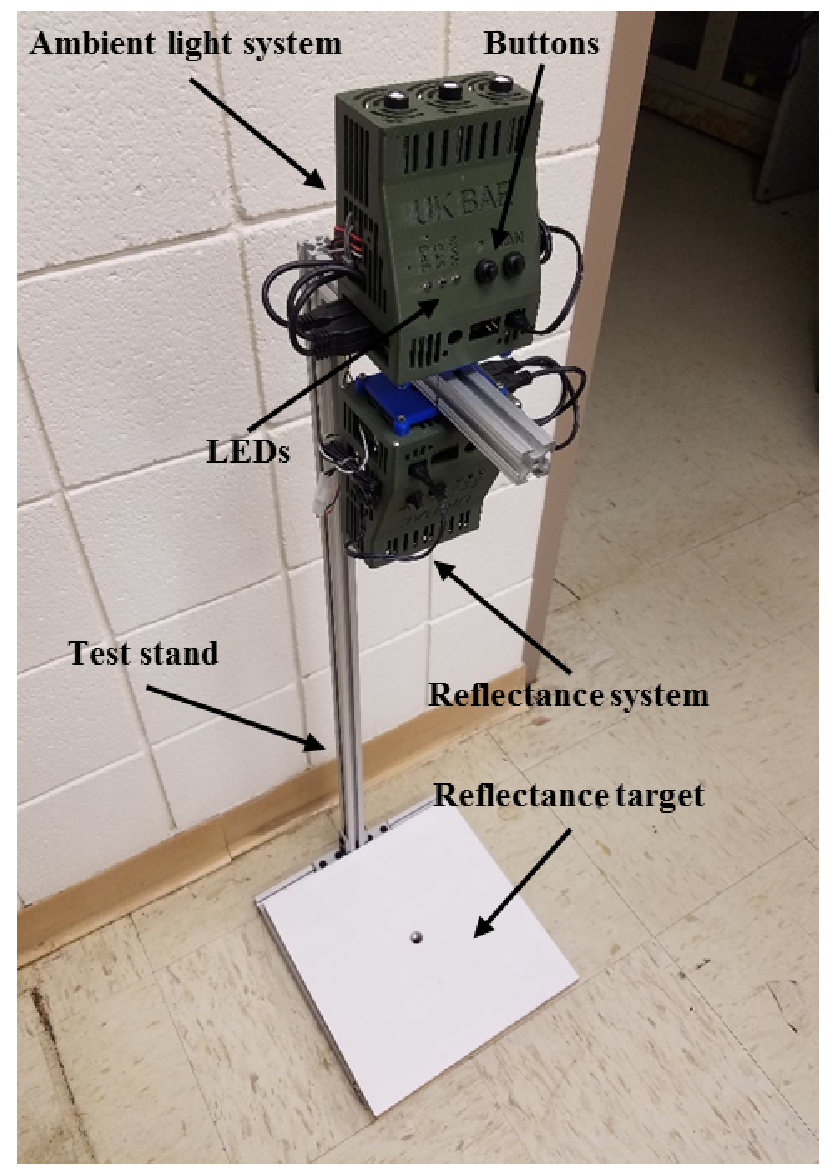

Figure 5: Stand for mounting spectrometers and placing reference target underneath sensors.

Table 2 and Figure 6 show the area covered (X and Y) at different heights above the reflectance target. In this setup, ambient light spectrometers were co-located on the top of reflectance spectrometers to mitigate variability in ambient light at the sensor location and the reflectance target. 


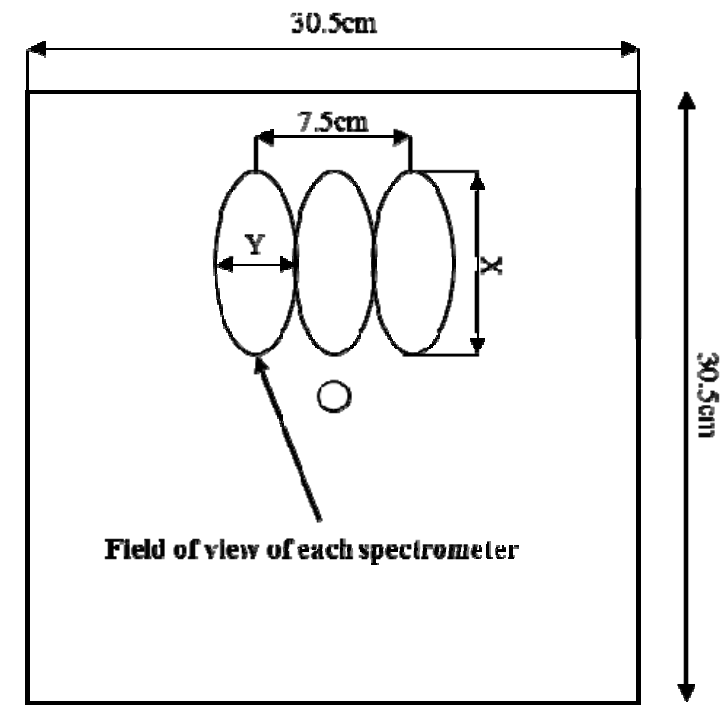

Figure 6: Schematic of area covered by a STS spectrometer on the reflectance target.

Table 2: Major (X) and minor (Y) axis dimensions of the FOV covered by the STS spectrometers using 74-DA lenses at different sensor heights above the reflectance target.

\begin{tabular}{ccccc}
\hline FOV & \multicolumn{4}{c}{ Height Above Target (m) } \\
\cline { 2 - 5 } Dimensions & 1 & 0.75 & 0.5 & 0.25 \\
\hline $\mathrm{Y}(\mathrm{m})$ & 0.04 & 0.03 & 0.02 & 0.01 \\
$\mathrm{X}(\mathrm{m})$ & 0.09 & 0.06 & 0.04 & 0.02 \\
\hline
\end{tabular}

\subsection{Integration Time}

In addition to ambient light conditions, integration time of the reflectance spectrometers was considered as an important parameter in reflectance measurements. Integration time is effectively a form of gain on the input signal - if set too high, the spectrometer will produce a saturated output, and if set too low, the output will lack sufficient detail for target classification. Doubling the integration time was assumed to have a similar effect of doubling the ambient light intensity. In this study, integration time for each reflectance spectrometer was manually adjusted to maximize the output signal without saturation in any wavelength during maximum ambient light intensity and keep constant during data collection. The integration times for the ambient light spectrometers were set according to the manufacturer recommendations to use the factory solar irradiance calibrations. Considering different integration times on each spectrometer, there were different time intervals for consecutive measurements for the ambient light and reflectance systems. Each spectral measurement reported was an average of 5 sequential measurements and there are three replications for each measurement, totaling 15 measurements on each spectrometer per measurement interval. Table 3 shows the integration time for each spectrometer and measurement interval for each system.

Table 3: Integration time and measurement interval for ambient light and reflectance spectrometers

\begin{tabular}{ccccc}
\hline \multirow{2}{*}{ Spectrometers } & \multicolumn{3}{c}{ Integration Time (ms) } & Measurement \\
\cline { 2 - 4 } & UV & VIS & NIR & Interval (s) \\
\hline Ambient light & 1000 & 180 & 1000 & 29 \\
Reflectance & 70 & 35 & 55 & 16 \\
\hline
\end{tabular}


Ideally, each reflectance measurement should be paired with an ambient light measurement taken at the same time. However, since integration times varied based on the spectrometer type and function, measurements were not temporally synchronized. To compensate for asychrnonous sampling, ambient light measurements were interpolated using two adjacent measurements and weighted according to the difference between the time of ambient light measurement and the time of reflectance measurement (ordinary kriging method).

\subsection{Calibration Equation}

Ambient light and sensor integration time were assumed to have a linear relationship with the raw measurement intensity from the reflectance system. Thus, compensating for ambient light and sensor integration time produced Equation 1, where the raw measurement intensity for each reflectance spectrometer was divided by the ambient light energy measured by the corresponding ambient light spectrometer and the integration time of the reflectance spectrometer.

$$
R_{\lambda}=\frac{M_{\lambda}}{S_{\lambda} \times t_{i}}
$$

Equation 1

Where:

$R$ was the compensated reflectance measurement from a sample (counts $\times \mu \mathrm{J}^{-1} \times \mathrm{ms}^{-1}$ )

$M$ was the raw measurement intensity of the reflectance spectrometer (counts)

$\mathrm{S}$ was the ambient light energy $(\mu \mathrm{J})$

$t_{i}$ was the integration time of the reflectance spectrometer (ms)

$\lambda$ was the specific wavelength (nm)

\subsection{Data collection}

Spectral data were collected during the 2017 Great American Eclipse at the Russellville-Logan County Airport in Russellville, KY on August 21, 2017. The spectrometer systems were mounted to the test frame and configured to record automatically between 10:26 am and 4:10 pm CST (Figure 7). Data collection encompassed the entire eclipse, including totality. 


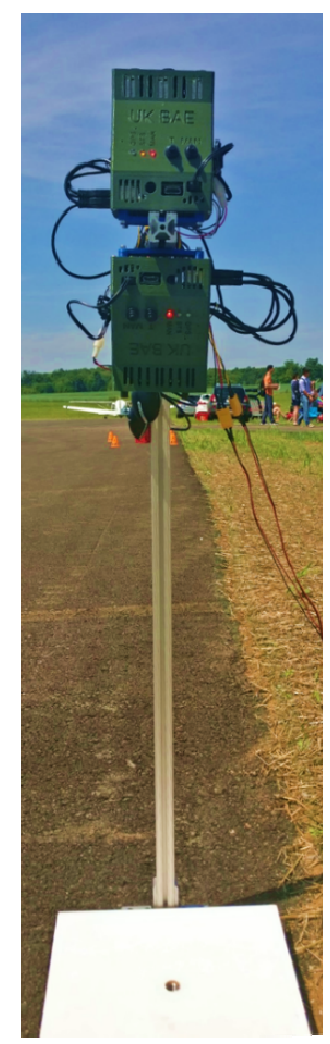

Figure 7: Data acquisition during Great American Eclipse 2017

Data processing and analysis were performed in MATLAB. A script was written to process data in four steps: 1) importing reflectance and ambient light data, 2) synchronizing reflectance and ambient light data, 3) applying the solar irradiance calibration to the ambient light data, and 4) compensating reflectance data with calibrated irradiance data and sensor integration time.

During this experiment, a weather station (HOBO U30; Onset Computer Corporation; Bourne, MA) was deployed to collect atmospheric data including ambient light, temperature, and humidity. Ambient light data from weather station was used to track the progress of the eclipse and as a reference for the spectrometers in the event any anomalous data were collected.

\section{Results and Discussion}

\subsection{Reference Target}

To benchmark the reflectance target, spectral responses were compared to a reference target which established $100 \%$ reflectance for the backscatter reflectance system. Figure 8 shows the relative reflectance of the reflectance target fell between $60 \%$ and $82 \%$ of the reference target. While not critical for this experiment, the reference target calibration would allow the data presented to subsequently be benchmarked to a reference standard if needed. It also indicated that the reflectance target did not uniformly reflect light across the wavelengths sampled during the experiment and tended to absorb NIR light at a higher rate than VIS light. 


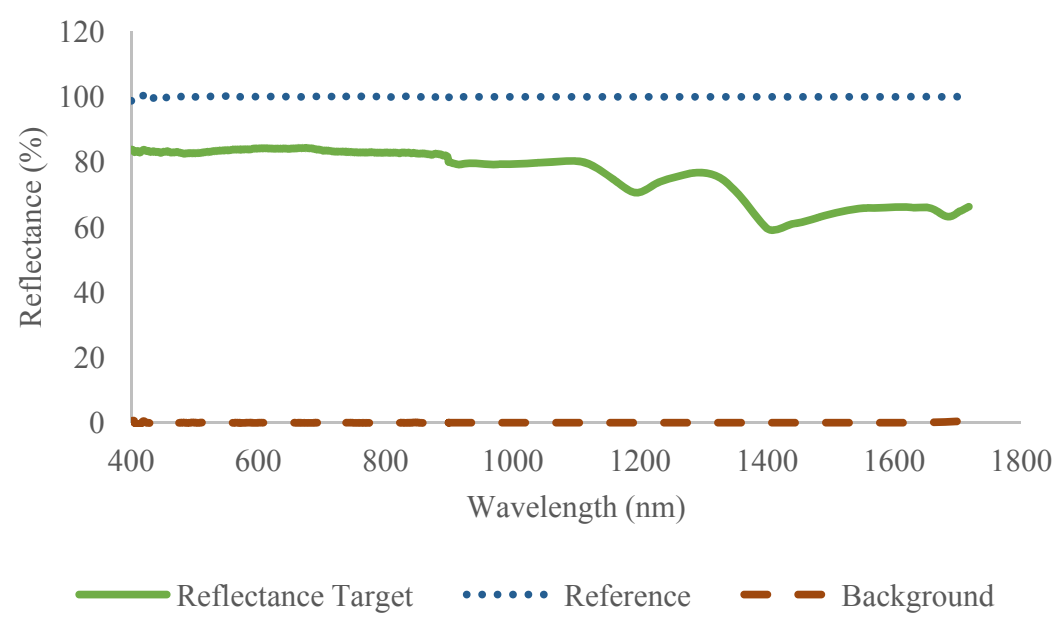

Figure 8: Reflectance target spectrum versus reference and background spectrums

\subsection{Ambient Light and Reflectance Measurements}

In Figure 9, the raw spectral data from the ambient light spectrometers can be seen in uncalibrated units of 14-bit integer counts. Each line represents an individual measurement and there were 413 measurements in total for each ambient light spectrometer. The variability in intensity for most of the wavelengths was high as a result ambient light changes during the eclipse. Low-intensity responses represent the measurements taken near totality. On the other hand, high intensity responses indicate the measurement when either the eclipse was just started partially or completely finished.
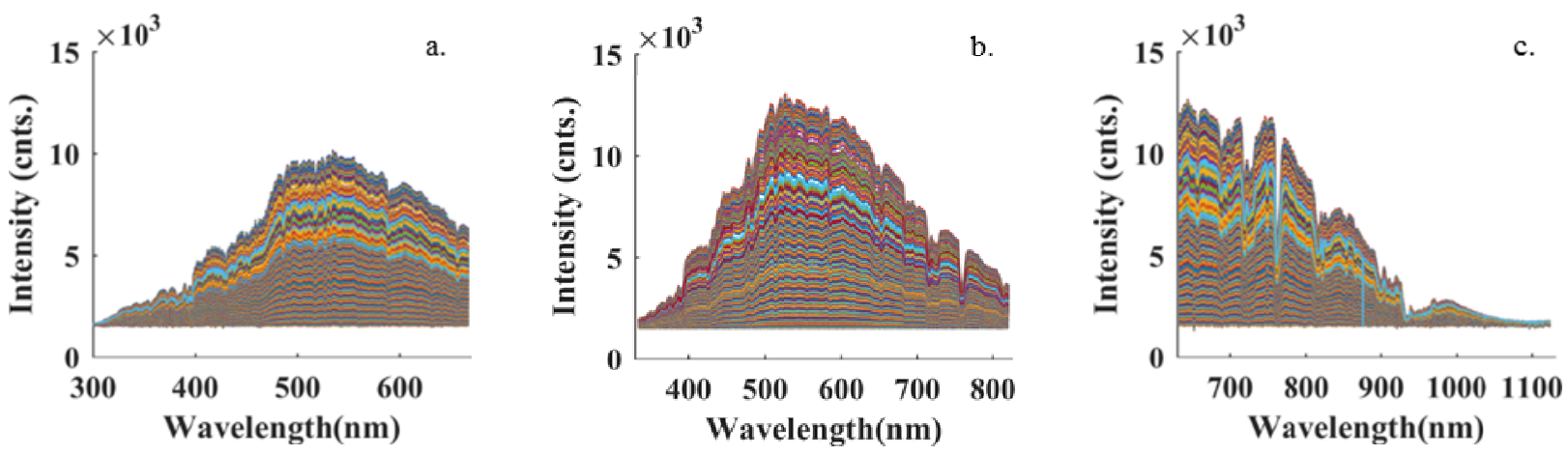

Figure 9: Raw ambient light measurements during the eclipse for a. UV; b. VIS; c. NIR spectrometers

Interpolating ambient light measurements to the reflectance measurement sampling interval and applying calibration coefficients produced spectral responses in units of microjoules (Figure 10). Applying the calibration coefficients removed much of the spectral variability inherent in the ambient light systems that occurs near the upper and lower wavelengths. The resulting spectral responses closely match each other in areas where there sampled wavelengths intersect with some minor variations. 

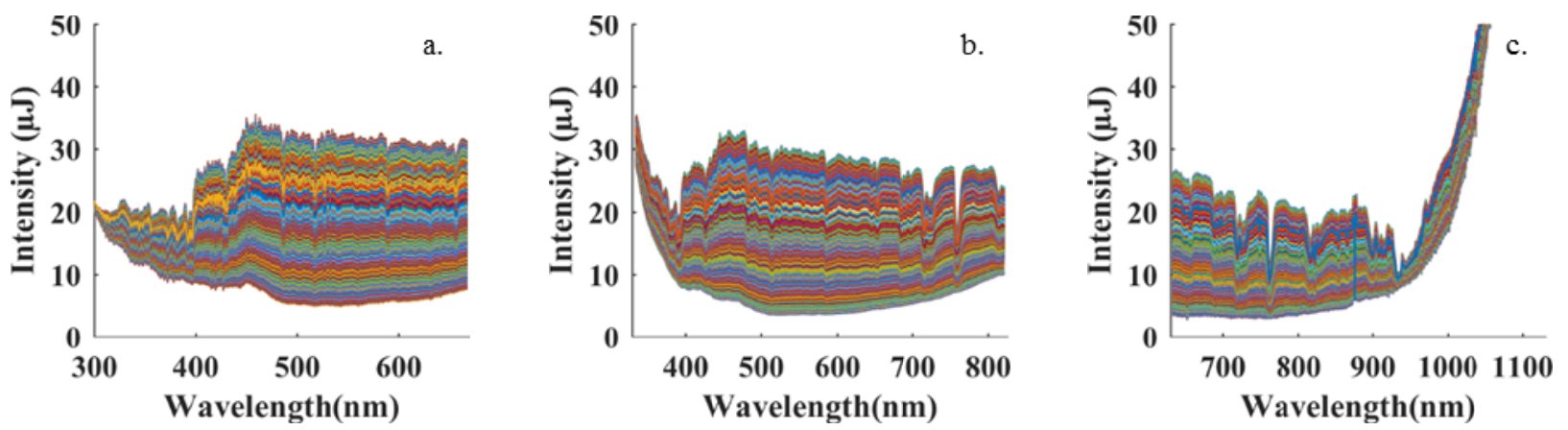

Figure 10: Calibrated ambient light measurements during the eclipse for a. UV; b. VIS; c. NIR spectrometer

The raw reflectance data are shown in Figure 11 exhbited similar responses as the raw ambient light measurements with similar variability in intensity due to varying ambient light intensity during the eclipse. Again, the lowest raw reflectance intensity occurred during totality, and the high-intensites represented measurements at the beginning or the end of the eclipse. The VIS reflectance spectrometer had a single "hot" pixel at $787.4 \mathrm{~nm}$ that should be ignored during subsequent analysis.
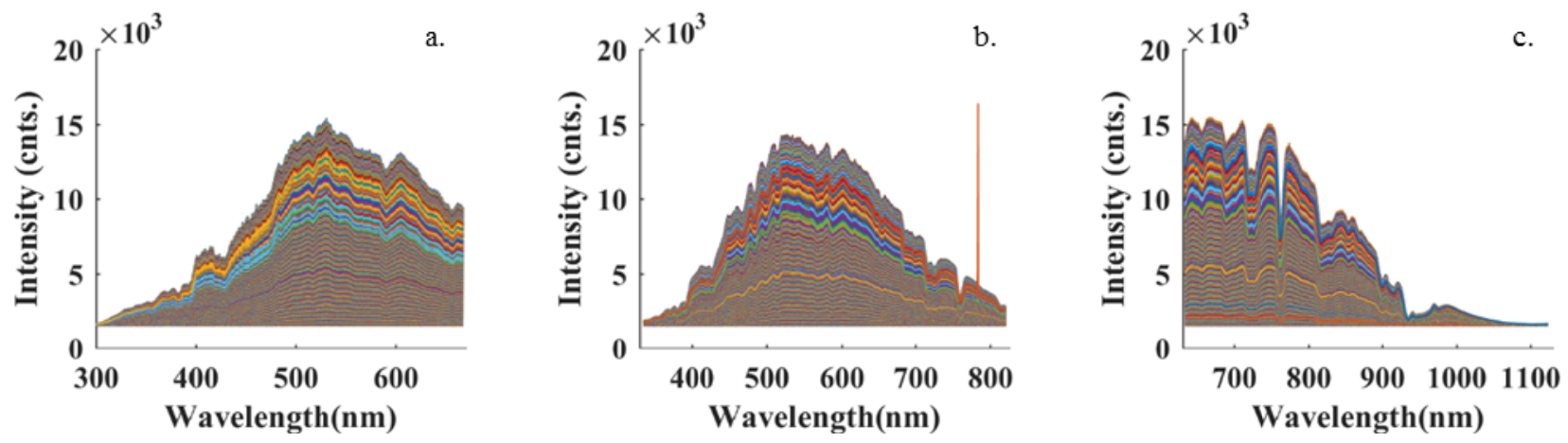

Figure 11: Raw reflected light measurements from a constant target during the eclipse for a. UV; b. VIS; c. NIR spectrometers

Calibrated ambient light and spectrometer integration times were combined with the raw reflectance intensity measurements using Equation 1 (Figure 12). The intensity unit changed accordingly to integer counts per microjoule of ambient light energy per millisecond of spectrometer integration time. The result of the compensation was a large reduction in variability centered around an average response with only a few outliers for each spectrometer. This meant that if a spectral measurement was taken from the reflectance target at a random ambient light condition, the compensated spectrum would have a high probability of being located near the average response. 

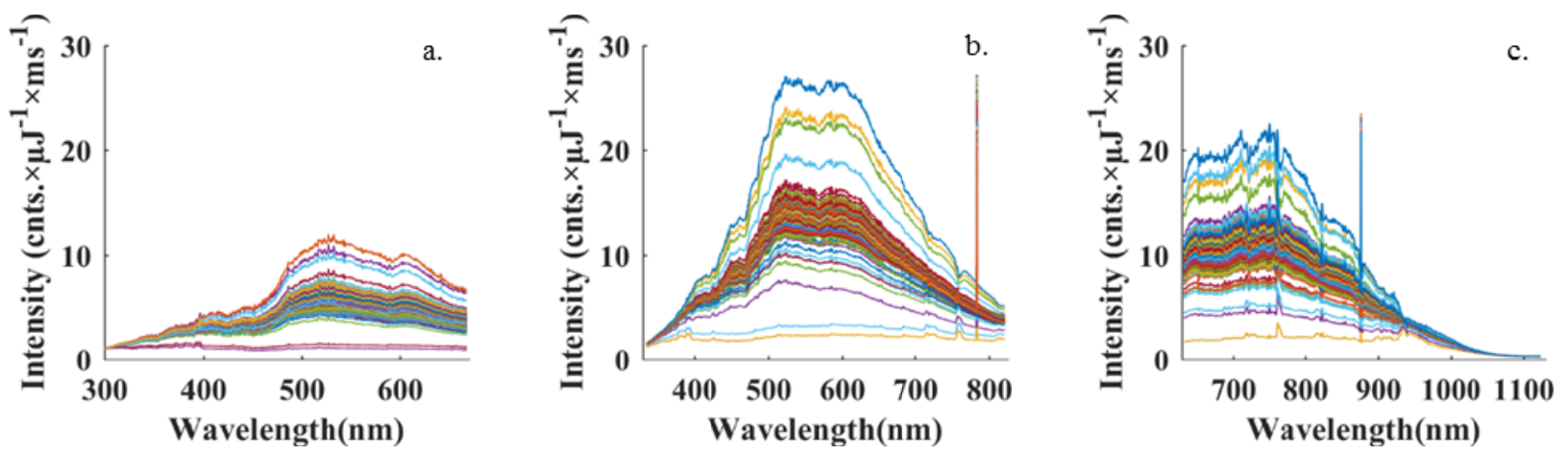

Figure 12: Compensated reflected light measurements from a constant target during the eclipse for a. UV; b. VIS; c. NIR spectrometers

\subsection{Calibration Evaluation}

Discrete probability density functions were used to assist with visualizing the reduction in variability due to ambient light conditions. One wavelength was selected for each spectrometer, which had the highest intensity variability in raw reflectance measurements. Figure 13 shows how the compensation process enhanced the probability for classifying the reflectance target over a range of values. It was desired to have approximately the same intensity for different measurements taken regardless of ambient light conditions. It can be observed that the intensity is highly scattered in raw relfectance measurements; whereas, after compensation, the probability is high only at one or two intensity groups. In another word, since the target is constant, the compensated reflectance intensity varied little over a wide range of ambient light conditions. When applied to all wavelengths, a unique signature for the target would be defined and it could be recognized using this signature, regardless of ambient light conditions.
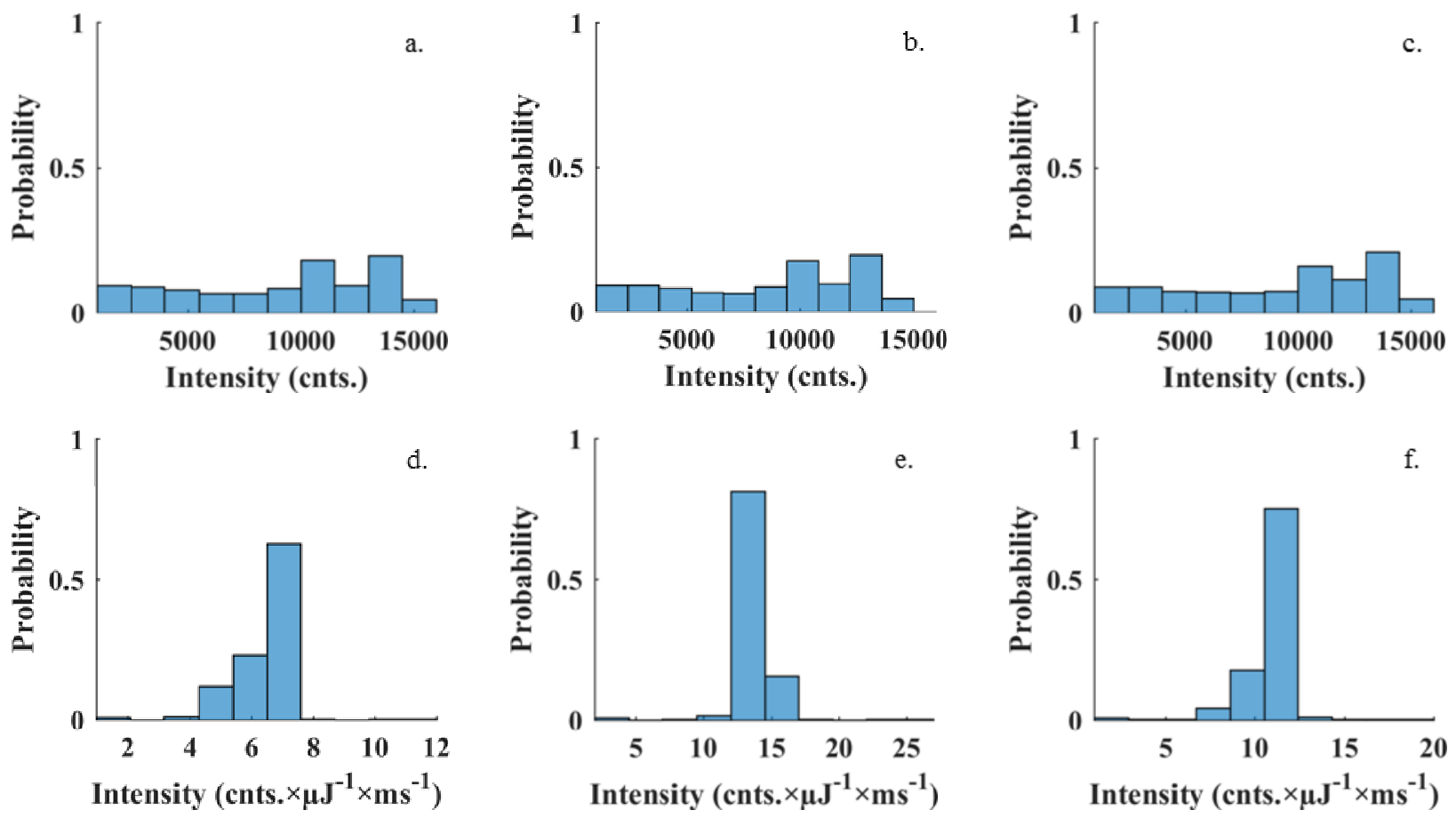

Figure 13: Histograms of the reflectance intensity from the constant target at the wavelength with the peak reflectance intensity: a. UV before compensation; b. VIS before compensation; c. NIR before compensation; d. UV after compensation; e. VIS after compensation; f. NIR after compensation 


\section{Conclusions}

In the first part of this study, a platform was developed to be deployed on a UAS to measure the reflectance intensity from a target. Two sets of portable STS spectrometers in three ranges of UV, VIS, and NIR were used along with a RPi to form a reflectance system and an ambient light system.in this platform. In the second part of this study, a method for compensating for ambient light conditions and sensor integration time was developed and tested during the 2017 Great American Eclipse. Results showed a large variability in reflected light intensity due to significant changes in sun radiance. Reflectance values were compensated using ambient light measurements and integration time. Compensated reflectance values showed a consistent spectral signature for measurements taken at different ambient light conditions. This method will useful for future field work where ambient light conditions cannot be controlled and the sensor integration time may need to be adjusted to optimize the sensitivity of the spectrometer. Future work should include testing the ability to classify different targets at varying ambient light conditions and to automatically adjust the integration time of each reflectance spectrometer based on previous measurements to maximize sensitivity.

\section{References}

[1] Kang, S., Shi, P., Pan, Y., Liang, Z., Hu, X., \& Zhang, J. Soil water distribution, uniformity and water-use efficiency under alternate furrow irrigation in arid areas. Irrigation Science, 19(4), 181-190. (2000)

[2] Perry, C. Efficient irrigation; inefficient communication; flawed recommendations. Irrigation and drainage, 56(4), 367-378. (2007)

[3] Yari, A., Madramootoo, C. A., Woods, S. A., \& Adamchuk, V. I. Performance evaluation of constant versus variable rate irrigation. Irrigation and drainage, 66(4), 501-509. (2017)

[4] O’Shaughnessy, S. A., Evett, S. R., \& Colaizzi, P. D. Dynamic prescription maps for site-specific variable rate irrigation of cotton. Agricultural Water Management, 159, 123-138. (2015)

[5] Buck, S., Nemati, M., \& Sunding, D. (2016). The Welfare Consequences of the 2015 California Drought Mandate: Evidence from New Results on Monthly Water Demand. Paper presented at the 2016 Annual Meeting, July 31August 2, 2016, Boston, Massachusetts.

[6] Pádua, L., Vanko, J., Hruška, J., et al. UAS, sensors, and data processing in agroforestry: a review towards practical applications. International Journal of Remote Sensing, 38(8-10), 2349-2391. (2017)

[7] Chrétien, L., Théau, J., \& Ménard, P. Wildlife multispecies remote sensing using visible and thermal infrared imagery acquired from an unmanned aerial vehicle (UAV). The International Archives of Photogrammetry, Remote Sensing and Spatial Information Sciences, 40(1), 241. (2015)

[8] Matese, A., Toscano, P., Di Gennaro, S. F., et al. Intercomparison of uav, aircraft and satellite remote sensing platforms for precision viticulture. Remote Sensing, 7(3), 2971-2990. (2015)

[9] Bajwa, S., \& Tian, L. Soil fertility characterization in agricultural fields using hyperspectral remote sensing. Transactions of the ASAE, 48(6), 2399-2406. (2005)

[10] Berni, J., Zarco-Tejada, P., Suárez, L., González-Dugo, V., \& Fereres, E. Remote sensing of vegetation from UAV platforms using lightweight multispectral and thermal imaging sensors. Int. Arch. Photogramm. Remote Sens. Spatial Inform. Sci, 38(6). (2009)

[11] Candiago, S., Remondino, F., De Giglio, M., Dubbini, M., \& Gattelli, M. Evaluating multispectral images and vegetation indices for precision farming applications from UAV images. Remote Sensing, 7(4), 4026-4047. (2015)

[12] Díaz-Varela, R. A., de la Rosa, R., León, L., \& Zarco-Tejada, P. J. High-resolution airborne UAV imagery to assess Olive tree crown parameters using 3D photo reconstruction: Application in breeding trials. Remote Sensing, 7(4), 4213-4232. (2015)

[13] Geipel, J., Link, J., \& Claupein, W. Combined spectral and spatial modeling of corn yield based on aerial images and crop surface models acquired with an unmanned aircraft system. Remote Sensing, 6(11), 10335-10355. (2014)

[14] Hung, C., Xu, Z., \& Sukkarieh, S. Feature learning based approach for weed classification using high resolution aerial images from a digital camera mounted on a uav. Remote Sensing, 6(12), 12037-12054. (2014)

[15] Koot, T. M. (2014). Weed detection with unmanned aerial vehicles in agricultural systems. (Master's Thesis), Wageningen University and Research Centre. 
[16] Xiang, H., \& Tian, L. Development of a low-cost agricultural remote sensing system based on an autonomous unmanned aerial vehicle (UAV). Biosystems engineering, 108(2), 174-190. (2011)

[17] Burkart, A., Cogliati, S., Schickling, A., \& Rascher, U. A novel UAV-based ultra-light weight spectrometer for field spectroscopy. IEEE sensors journal, 14(1), 62-67. (2014)

[18] Von Bueren, S., Burkart, A., Hueni, A., Rascher, U., Tuohy, M., \& Yule, I. Deploying four optical UAV-based sensors over grassland: challenges and limitations. Biogeosciences, 12(1), 163-175. (2015)

[19] Tsouvaltsidis, C., Al Salem, N. Z., Benari, G., Vrekalic, D., \& Quine, B. Remote Spectral Imaging Using a Low Cost UAV System. The International Archives of Photogrammetry, Remote Sensing and Spatial Information Sciences, 40(1), 25. (2015)

[20] Zeng, C., King, D. J., Richardson, M., \& Shan, B. Fusion of Multispectral Imagery and Spectrometer Data in UAV Remote Sensing. Remote Sensing, 9(7), 696. (2017)

[21] Eismann, M. T. (2012). Hyperspectral Remote Sensing (pp. 417-450): SPIE Bellingham.

[22] Cocks, T., Jenssen, R., Stewart, A., Wilson, I., \& Shields, T. (1998). The HyMapTM airborne hyperspectral sensor: the system, calibration and performance. Paper presented at the Proceedings of the 1st EARSeL workshop on Imaging Spectroscopy.

[23] Hamidisepehr, A., Sama, M. P., Turner, A. P., \& Wendroth, O. O. A Method for Reflectance Index Wavelength Selection from Moisture-Controlled Soil and Crop Residue Samples. Transactions of the ASABE, 60(5), 14791487. (2017) 